\title{
ON STRONG LIFTING COMPACTNESS, WITH APPLICATIONS TO TOPOLOGICAL VECTOR SPACES
}

\author{
A. G. A. G. BABIKER, G. HELLER and W. STRAUSS
}

(Received 1 September 1984)

Communicated by W. Moran

\begin{abstract}
The notion of strong lifting compactness is introduced for completely regular Hausdorff spaces, and its structural properties, as well as its relationship to the strong lifting, to measure compactness, and to lifting compactness, are discussed. For metrizable locally convex spaces under their weak topology, strong lifting compactness is characterized by a list of conditions which are either measure theoretical or topological in their nature, and from which it can be seen that strong lifting compactness is the strong counterpart of measure compactness in that case.
\end{abstract}

1980 Mathematics subject classification (Amer. Math. Soc.): 28 A 51.

\section{Introduction}

The notions of lifting compact spaces and lifting compact maps from a probability space into a completely regular Hausdorff space $T$ were introduced in [3] and [4]. For any lifting-compact map $\varphi$ and any lifting on the space of all bounded measurable functions on the underlying probability space, there is an associated 'lifted function' $\rho^{\prime}(\varphi)$ of $\varphi$ taking values in $T ; \rho^{\prime}(\varphi)$ is Borel measurable and satisfies the condition

$$
\rho(h \circ \varphi)=h \circ \rho^{\prime}(\varphi),
$$

for all $h \in \mathscr{C}^{b}(T)$, the space of all bounded continuous functions on $T$ [3, page 237]. If $T$ has a metrizable compactification, a stronger version of (1) is valid. The weak equivalence of $\varphi$ and $\rho^{\prime}(\varphi)$ given by (1) becomes a strong equivalence in the

(C) 1986 Australian Mathematical Society $0263-6115 / 86 \$ A 2.00+0.00$ 
sense that

$$
\rho^{\prime}(\varphi)=\varphi \quad \mu \text {-a.e. }
$$

for $h \in \mathscr{C}^{b}(T)$ [3, page 244].

In this paper we study those lifting compact maps (respectively spaces) for which the stronger condition (2) is valid, and which we call strongly lifting compact maps (respectively spaces).

Section 1 contains notation and preliminary material. In Section 2 we show that submetrizability is a sufficient condition for strong lifting compactness, and we relate strong lifting compactness to the notion of the strong lifting.

In Section 3 we first study structural properties of strong lifting compactness. This provides a basis on which we can check its relationships with measure compactness, strong measure compactness, and lifting compactness, as well as to the strong lifting property. We then show that strong lifting compactness is a local property.

The case of a metrizable locally convex topological vector space $X$ under the weak topology is studied separately in Section 4 . The first theorem characterizes a strongly lifting compact $X$-valued map, for instance, by the property that it agrees a.e. with a Bochner measurable map.

This leads immediately to a description of strongly lifting compact spaces $X$ in Theorem 4.3.

A topological characterization is given in Theorem 4.7: $X$ is strongly lifting compact if and only if it is measure compact and can be continuously linearly injected into $\mathbb{R}^{N}$. Example 4.10(iii) shows that this does not imply separability of $X$.

\section{Preliminaries}

Throughout, $T$ denotes a completely regular Hausdorff space, and $\mathscr{C}^{b}(T)$ is the space of all bounded continuous real valued functions on $T .(\Omega, \Sigma, \mu)$ denotes a complete probability space, and $\mathscr{L}^{\infty}(\Omega, \Sigma, \mu)$, or just $\mathscr{L}^{\infty}(\mu)$, is the space of all bounded $\Sigma$-measurable functions on $\Omega$. By a measure we always mean a positive, countably additive, real valued set function on the $\sigma$-field involved. The $\sigma$-field of Baire (respectively Borel) sets over $T, \mathscr{B}_{T}$ (respectively $\mathscr{B}_{T}^{0}$ ), is the one generated by all functions in $\mathscr{C}^{b}(T)$ (respectively by all open subsets of $T$ ). Let $\nu$ be a measure on $\left(T, \mathscr{B}_{T}\right)$ (respectively $\left(T, \mathscr{B}_{T}^{0}\right)$ ). Then $\tilde{\mathscr{B}}_{T}$ (respectively $\tilde{\mathscr{B}}_{T}^{0}$ ) denotes the Carathéodory completion with respect to $\nu$, and $\left(T, \mathscr{B}_{T}, \nu\right)$ is called completion regular if $\mathscr{B}_{T}^{0} \subset \tilde{\mathscr{B}}_{T} . T$ itself is completion regular if this holds true for every measure on $\left(T, \mathscr{B}_{T}\right)$. 
A function $\varphi^{*}$ from the measurable space $(\Omega, \Sigma)$ will be called Baire (respectively Borel) measurable if it is measurable with respect to the Baire (respectively Borel) $\sigma$-field on $T$.

All unexplained topological measure theoretic notions will be those of [10], [12], and [14]. Those concerning lifting theory can be found in [8].

Let $\nu$ be a measure on $\left(T, \mathscr{B}_{T}\right)$.

A lifting $\rho$ for $\left(T, \tilde{\mathscr{B}}_{T}, \tilde{\nu}\right)$ is called an almost strong Baire lifting if there is a $\tilde{\nu}$-negligible subset $N$ of $T$ such that $\rho(h)(t)=h(t)$ for every $h \in \mathscr{C}^{b}(T)$ and $t \in T \backslash N$. Babiker and Strauss [2] have shown that $\nu$ is $\tau$-additive, and that $\left(T, \mathscr{B}_{T}, \nu\right)$ is completion regular, whenever there is an almost strong Baire lifting for $\left(T, \tilde{\mathscr{B}}_{T}, \tilde{\boldsymbol{v}}\right)$.

Let $\rho$ be a lifting for $\mathscr{L}^{\infty}(\mu)$. Any Baire measurable map $\varphi: \Omega \rightarrow T$ induces a Borel measurable map $\rho^{\prime}(\varphi)$ from $\Omega$ into $\beta T$, the Stone-Cech compactification of $T$, defined by the formula

$$
h \circ \rho^{\prime}(\varphi)=\rho(h \circ \varphi), \quad h \in \mathscr{C}^{b}(T) .
$$

We call $\varphi$ lifting compact if $\rho^{\prime}(\varphi)(\omega) \in T \mu$-a.e. for every lifting $\rho$ of $\mathscr{L}^{\infty}(\omega)$. The following fact will be frequently used without explicit mention.

If $\varphi: \Omega \rightarrow T$ is such that for any lifting $\rho$ of $\mathscr{L}^{\infty}(\mu)$, there is a map $\psi_{\rho}: \Omega_{0} \rightarrow T$ for some $\Omega_{0} \in \Sigma$ with $\mu\left(\Omega_{0}\right)=1$ which has the property that $h \circ \psi_{\rho} \in \mathscr{L}^{\infty}(\mu)$ and

$$
\rho(h \circ \varphi)(\omega)=h \circ \psi_{\rho}(\omega), \quad \omega \in \Omega_{0}, h \in H,
$$

where $H \subset \mathscr{C}^{b}(T)$ generates the topology of $T$, then $\varphi$ is Baire measurable, and we have

$$
\rho(h \circ \varphi)(\omega)=h \circ \psi_{p}(\omega), \quad \omega \in \Omega_{0}, h \in \mathscr{C}^{b}(T) .
$$

This implies that $\left.\rho^{\prime}(\varphi)\right|_{\Omega_{0}} \equiv \psi_{\rho}$, so that $\varphi$ is lifting compact (cf. [3, page 242]).

\section{Strong lifting compactness}

A Baire measurable map $\varphi$ from $\Omega$ into $T$ is said to be strongly lifting compact if, for any lifting $\rho$ of $\mathscr{L}^{\infty}(\mu)$, there is a set $\Omega_{0} \in \Sigma$ with $\mu\left(\Omega_{0}\right)=1$ such that

$$
\rho(h \circ \varphi)(\omega)=(h \circ \varphi)(\omega), \quad h \in \mathscr{C}^{b}(T), \omega \in \Omega_{0},
$$

i.e. $\varphi$ is strongly lifting compact if and only if $\varphi$ is lifting compact, and for all liftings $\rho$ of $\mathscr{L}^{\infty}(\mu)$, we have $\rho^{\prime}(\varphi)=\varphi \mu$-a.e.

Strong lifting compactness is stable under composition with continuous maps.

2.1. TheOREM. Let $\varphi: \Omega \rightarrow T$ be strongly lifting compact, and let $r$ be a continuous map from $T$ into a completely regular Hausdorff space $S$. Then $r \circ \varphi$ is strongly lifting compact. 
Proof. Let $\rho$ be a lifting of $\mathscr{L}^{\infty}(\mu)$, and let $\Omega_{0} \in \Sigma$ such that $\mu\left(\Omega_{0}\right)=1$ and

$$
\left.\rho^{\prime}(\varphi)\right|_{\Omega_{0}}=\left.\varphi\right|_{\Omega_{0}}
$$

If $h \in \mathscr{C}^{b}(S)$, we have

$$
\rho(h \circ r \circ \varphi)(\omega)=h \circ r \circ \rho^{\prime}(\varphi)(\omega)=h \circ r \circ \varphi(\omega), \quad \omega \in \Omega_{0} .
$$

Therefore $\rho^{\prime}(r \circ \varphi)=r \circ \varphi \mu$-a.e., as desired.

The following theorem gives a sufficient condition for strong lifting compactness.

2.2. THEOREM. Suppose that $T$ is lifting compact and that $\varphi$ is a Baire measurable map from $\Omega$ into $T$. If $\mathrm{cl}_{T}(\varphi(\Omega)$ ), the closure of $\varphi(\Omega)$ in $T$, has a metrizable subtopology, then $\varphi$ is strongly lifting compact.

Proof. Since $T$ is lifting compact, the image measure $\varphi(\mu)$ of $\mu$ under $\varphi$ is $\tau$-additive and therefore has non-empty support $S$ in $T$. Clearly $S \subset \operatorname{cl}_{T}(\varphi(\Omega)$ ), and $\rho^{\prime}(\varphi)(\omega) \in S \mu$-a.e. Since $\mathscr{C}^{b}(T)$ generates the topology of $\mathrm{cl}_{T}(\varphi(\Omega))$, we see that $\varphi$, as a map from $\Omega$ to $\mathrm{cl}_{T}(\varphi(\Omega))$, is lifting compact; so we may assume $\varphi(\Omega)$ to be dense in $T$.

Now let $d$ be a continuous metric on $T$. Then $\varphi(\mu)$ induces a $\tau$-additive Baire measure on $(T, d)$ having non-empty separable support $S_{0}$ which clearly contains $S$.

Let $\left(h_{n}\right)$ be a sequence in $\mathscr{C}^{b}(T, d) \subset \mathscr{C}^{b}(T)$ which separates the points of $S_{0}$, and let $\Omega_{0} \in \Sigma$ be such that $\mu\left(\Omega_{0}\right)=1, \rho^{\prime}(\varphi)\left(\Omega_{0}\right) \subset S \subset S_{0}$, and

$$
h_{n} \circ \varphi(\omega)=\rho\left(h_{n} \circ \varphi\right)(\omega)=h_{n} \circ \rho^{\prime}(\varphi)(\omega), \quad n \in \mathbb{N}, \omega \in \Omega_{0} .
$$

Since $\left(h_{n}\right)$ separates the points of $S_{0}$, it follows that

$$
\varphi=\rho^{\prime}(\varphi) \quad \mu \text {-a.e. }
$$

Strong lifting compactness of a map $\varphi$ requires more than the existence of strong liftings for the induced measure space on $T$. For a Baire measurable map $\varphi: \Omega \rightarrow T$, let $\lambda$ be the image measure of $\mu$ under $\varphi$ on the induced $\sigma$-field

$$
\mathscr{B}_{\Sigma}:=\left\{A \subset T \mid \bar{\varphi}^{1}(A) \in \Sigma\right\} \supset \mathscr{B}_{T} .
$$

We then have the following necessary condition for strong lifting compactness.

2.3. THEOREM. If $\varphi$ is strongly lifting compact, then every lifting $\theta$ of $\mathscr{L}^{\infty}(\lambda)$ is almost strong; that is, there is $N \in \mathscr{B}_{\Sigma}$ with $\lambda(N)=0$ such that

$$
\theta(h)(t)=h(t), \quad h \in \mathscr{C}^{b}(T), t \in T \backslash N .
$$

Proof. Let $\theta$ be a lifting of $\mathscr{L}^{\infty}(\lambda)$ and put

$$
H_{0}:=\left\{f \circ \varphi \mid f \in \mathscr{L}^{\infty}(\lambda)\right\}
$$


Let

$$
H:=\left\{g \in \mathscr{L}^{\infty}(\mu) \mid g=g^{\prime} \mu \text {-a.e. for some } g^{\prime} \in H_{0}\right\} \text {. }
$$

Then $H$ is an admissible subalgebra of $\mathscr{L}^{\infty}(\mu)$ in the sense of [8, Chapter II, Section 1, Definition 1]). Define $\rho_{1}$ by

$$
\rho_{1}(g):=\theta(f) \circ \varphi
$$

where $g \in H$ and $g=f \circ \varphi \mu$-a.e. Then $\rho_{1}$ is an unambiguously defined lifting on $H$ and can be extended to a lifting $\rho$ on $\mathscr{L}^{\infty}(\mu)([8$, Chapter IV, Section 2, Theorem 4]). Since $\varphi$ is strongly lifting compact, there is $\Omega_{0} \in \Sigma$ with $\mu\left(\Omega_{0}\right)=1$ and

But then

$$
\rho(h \circ \varphi)(\omega)=h \circ \varphi(\omega), \quad h \in \mathscr{C}^{b}(T), \omega \in \Omega_{0}
$$

$$
\theta(h)(t)=h(t), \quad h \in \mathscr{C}^{b}(T), t \in \varphi\left(\Omega_{0}\right),
$$

whence $\theta$ is almost strong, since $\varphi\left(\Omega_{0}\right) \in \mathscr{B}_{\Sigma}$ and $\lambda\left(\varphi\left(\Omega_{0}\right)\right)=1$.

We do not know whether the requirement in 2.3 for liftings of $\mathscr{L}^{\infty}(\lambda)$ is sufficient for the strong lifting compactness of $\varphi$ in general. This question is answered positively for metrizable linear spaces in Section 4. Here we shall demonstrate the converse of 2.3 in the case when $\varphi$ is injective.

2.4. THEOREM. If $\varphi$ is an injective Baire measurable map, and if every lifting of $\mathscr{L}^{\infty}(\lambda)$ is almost strong, then $\varphi$ is strongly lifting compact.

Proof. Since $\varphi(\Omega) \in \mathscr{B}_{\Sigma}$ and $\lambda(\varphi(\Omega))=1$, we may assume that $\varphi$ is bijective. Clearly then $\varphi^{-1}$ is measurable with respect to $\mathscr{B}_{\Sigma}$ and $\Sigma$. If $\rho$ is a lifting for $\mathscr{L}^{\infty}(\mu)$, then a lifting $\theta$ for $\mathscr{L}^{\infty}(\mu)$ may be defined by

$$
\boldsymbol{\theta}(f):=\rho(f \circ \varphi) \circ \varphi^{-1}, \quad f \in \mathscr{L}^{\infty}(\lambda) .
$$

By assumption, there is a set $A_{0} \in \mathscr{B}_{\Sigma}$ with $\lambda\left(A_{0}\right)=1$ and

$$
\theta(h)(t)=h(t), \quad h \in \mathscr{C}^{b}(T), t \in A_{0} \text {. }
$$

But then

$$
\begin{aligned}
h \circ \varphi(\omega)=\theta(h)(\varphi(\omega))=\rho(h \circ \varphi)(\omega)=h \circ \rho^{\prime}(\rho)(\omega), & \\
h & \in \mathscr{C}^{b}(T), \omega \in \varphi^{-1}\left(A_{0}\right) .
\end{aligned}
$$

Since $\mu\left(\varphi^{-1}\left(A_{0}\right)\right)=1$, it follows that $\varphi=\rho^{\prime}(\varphi) \mu$-a.e.

\section{Strongly lifting compact spaces}

$T$ is called strongly lifting compact if, for every complete probability space $(\Omega, \Sigma, \mu)$, every Baire measurable map $\varphi: \Omega \rightarrow T$ is strongly lifting compact.

It is clear that every subspace of a compact metric space is strongly lifting compact. Moreover, this holds true for every strongly lifting compact space. 


\subsection{THEOREM.}

(i) Every subset of a strongly lifting compact space is strongly lifting compact.

(ii) Countable unions of strongly lifting compact Baire subsets of a completely regular Hausdorff space T are strongly lifting compact.

(iii) Countable products of strongly lifting compact spaces are strongly lifting compact.

(iv) If $T$ is strongly lifting compact, and if $r: T \rightarrow S$ is a continuous surjection which admits a Baire measurable section, then $S$ is strongly lifting compact.

Proof. (i) Let $S \subset T$, and let $\varphi: \Omega \rightarrow S$ be Baire measurable. Then $\imath^{\circ} \varphi$ : $\Omega \rightarrow T$ is strongly lifting compact where ı denotes the natural injection from $S$ into $T$. If $\rho$ is a lifting for $\mathscr{L}^{\infty}(\mu)$, then there is $\Omega_{0} \in \Sigma$ with $\mu\left(\Omega_{0}\right)=1$ such that

$$
h \circ \rho^{\prime}(\iota \circ \varphi)(\omega)=h \circ \iota \circ \varphi(\omega)=\left.h\right|_{S} \circ \varphi(\omega), \quad h \in \mathscr{C}^{b}(T), \omega \in \Omega_{0} .
$$

Since the family $\left\{\left.h\right|_{S} \mid h \in \mathscr{C}^{b}(T)\right\}$ generates the topology of $S$, it follows that

$$
h \circ \rho^{\prime}(\rho)(\omega)=h \circ \varphi(\omega), \quad \omega \in \Omega_{0}, h \in \mathscr{C}^{b}(S) .
$$

Hence $\left.\rho^{\prime}(\varphi)\right|_{\Omega_{0}}=\left.\varphi\right|_{\Omega_{0}}$.

(ii) Let $\left\{S_{n} \mid n \in N\right\} \subset \mathscr{B}_{T}$, where each $S_{n}$ is strongly lifting compact. By (i) we may assume that $S_{n} \cap S_{m}=\varnothing$ for $n \neq m$.

Let $\varphi: \Omega \rightarrow S:=\cup S_{n}$ be Baire measurable, and let $\rho$ be a lifting of $\mathscr{L}^{\infty}(\mu)$. For each $n$, let $\Omega_{n}:=\varphi^{-1}\left(S_{n}\right) \cap \rho\left(\varphi^{-1}\left(S_{n}\right)\right)$, and let $\varphi_{n}:=\left.\varphi\right|_{\Omega_{n}}$. Each $f \in$ $\mathscr{L}^{\infty}\left(\left.\mu\right|_{\Omega_{n}}\right)$ can be extended trivially to an element $\bar{f} \in \mathscr{L}^{\infty}(\mu)$ which vanishes outside $\Omega_{n}$. Clearly the function $\rho_{n}$, defined by $\rho_{n}(f):=\left.\rho(\bar{f})\right|_{\Omega_{n}}$ for $f \in \mathscr{L}^{\infty}\left(\left.\mu\right|_{\Omega_{n}}\right)$ is a lifting for $\mathscr{L}^{\infty}\left(\left.\mu\right|_{\Omega_{n}}\right)$. Since $S_{n}$ is strongly lifting compact, there is $\Omega_{n}^{\prime} \in \Sigma \cap \Omega_{n}$ with $\mu\left(\Omega_{n}^{\prime}\right)=\mu\left(\Omega_{n}\right)$ and $\rho_{n}\left(h \circ \varphi_{n}\right)(\omega)=h \circ \varphi_{n}(\omega)$ for all $\omega \in \Omega_{n}^{\prime}$ and $h \in$ $\mathscr{C}^{b}\left(S_{n}\right)$. Now let $\Omega_{0}:=\cup \Omega_{n}^{\prime} \in \Sigma$. Then $\mu\left(\Omega_{0}\right)=1$, and, for $h \in \mathscr{C}^{b}(S)$ and $\omega \in \Omega_{0}$, we have

$\rho(h \circ \varphi)(\omega)=\left.\sum \rho(h \circ \varphi)\right|_{\Omega_{n}^{\prime}}(\omega)=\sum \rho_{n}\left(h \circ \varphi_{n}\right)(\omega)=\sum h \circ \varphi_{n}(\omega)=h \circ \varphi(\omega)$. Therefore $\left.\rho^{\prime}(\varphi)\right|_{\Omega_{0}}=\left.\varphi\right|_{\Omega_{0}}$.

(iii) Let $\varphi: \Omega \rightarrow T:=\prod_{n \in \mathrm{N}} T_{n}$ be Baire measurable, where each $T_{n}$ is strongly lifting compact. If $p_{n}$ is the canonical projection of $T$ onto $T_{n}$, then $\varphi_{n}:=p_{n} \circ \varphi$ is Baire measurable. Let $\rho$ be a lifting of $\mathscr{L}^{\infty}(\mu)$. By assumption there exists $\Omega_{n} \in \Sigma$ such that $\mu\left(\Omega_{n}\right)=1$, and such that, for $h_{n} \in \mathscr{C}^{b}\left(T_{n}\right)$, we have

$$
\rho\left(h_{n} \circ \varphi_{n}\right)=h_{n} \circ \varphi_{n} \quad \text { on } \Omega_{n} \text { for all } n \text {. }
$$

The family $H$ of all functions $h_{n} \circ \varphi_{n}\left(h_{n} \in \mathscr{C}^{b}\left(T_{n}\right)\right)$ generates the topology of $T$. It we put $\Omega_{0}:=\cap \Omega_{n}$, then $\Omega_{0} \in \Sigma, \mu\left(\Omega_{0}\right)=1$, and $\rho(h \circ \varphi)=h \circ \varphi$ on $\Omega_{0}$ for all $h \in H$, whence $\varphi$ is strongly lifting compact.

(iv) Let $S=r(T)$, where $r: T \rightarrow S$ is a continuous map admitting a Baire measurable section $s: S \rightarrow T$. Then if $\varphi: \Omega \rightarrow S$ is Baire measurable, so also is $\psi:=s \circ \varphi: \Omega \rightarrow T$. Now if $\rho$ is a lifting of $\mathscr{L}^{\infty}(\mu)$, and if $\Omega_{0} \in \Sigma$ with $\mu\left(\Omega_{0}\right)=1$ 
is such that

$$
\rho(g \circ \psi)(\omega)=g \circ \psi(\omega), \quad g \in \mathscr{C}^{b}(T), \omega \in \Omega_{0},
$$

then we get the desired assertion that

$$
\begin{aligned}
\rho(h \circ \varphi)(\omega) & =\rho((h \circ r) \circ \psi)(\omega) \\
& =h \circ r \circ \psi(\omega)=h \circ \varphi(\omega), \quad h \in \mathscr{C}^{b}(S), \omega \in \Omega_{0} .
\end{aligned}
$$

3.2. Remarks. (i) Let $\lambda$ be any topological measure on $T$, i.e. a countably additive measure on a complete $\sigma$-field $\Theta$ of subsets on $T$ which contains $\mathscr{B}_{T}$. By applying 2.3 to $(\Omega, \Sigma, \mu)=(T, \Theta, \lambda)$ and to $\varphi=$ the identity map of $T$, it follows that if $T$ is strongly lifting compact, then for every topological measure $\lambda$ on $T$, every lifting of $\mathscr{L}^{\infty}(T, \Theta, \lambda)$ is almost strong. We do not know whether the converse is true in general, but in Section 4 we show that the converse holds for metrizable linear spaces under the weak topology.

(ii) The condition that the map $r$ in Theorem 3.1(iv) have a Baire measurable section cannot be dropped. For let $T$ be any compact space whose cardinal is non-measurable, and which admits a Radon measure $\nu$ with full support for which no strong lifting exists (see [11]). Let $D$ be the discrete space with the same cardinality as $T$ and $r$ any bijection from $D$ onto $T$. From $2.2, D$ is strongly lifting compact, while from Remark (i), $T$ is not, i.e. a continuous bijective map need not necessarily be strongly lifting compact.

(iii) The support of a $\tau$-additive Baire measure on a metric space can be homeomorphically embedded into a Polish space, namely its completion with respect to the induced metric. From this, one easily concludes that for a general metric space $T$, strong lifting compactness is equivalent to measure compactness, and this in turn is equivalent to the condition that every closed discrete subspace of $T$ have non-measurable cardinal. Clearly this is not true in general, since measure compactness does not even imply lifting compactness, a condition which lies strictly between strong measure compactness and measure compactness (see A. Bellow [2]). V. Losert's counterexample in [11] together with 2.3 shows that neither lifting compactness nor strong measure compactness imply strong lifting compactness. Nor does strong lifting compactness imply strong measure compactness, as witnessed by the standard Lebesgue non-measurable subset of $[0,1]$.

The following result shows that local strong lifting compactness implies global strong lifting compactness in the case when $T$ is measure compact.

3.3. THEOREM. Let $T$ be measure compact and suppose that every point in $T$ has a neighborhood which is strongly lifting compact. Then T is strongly lifting compact.

Proof. By assumption we may cover $T$ by a family $\mathscr{F}$ of strongly lifting compact cozero subsets which, by 3.1(ii), we may assume to be directed upwards. 
If $\varphi: \Omega \rightarrow T$ is Baire measurable, then the image measure $\varphi(\mu)$ is $\tau$-additive since $T$ is measure compact. So we may choose an increasing sequence $\left(U_{n}\right)$ in $\mathscr{F}$ such that

$$
\varphi(\mu)\left(T \backslash \bigcup U_{n}\right)=0,
$$

and it is sufficient to show that $U U_{n}$ is strongly lifting compact. The result now follows from 3.1(ii).

3.4. COROLlaRY. Every locally metrizable measure compact space is strongly lifting compact.

\section{The case of metrizable locally convex topological vector spaces}

Throughout, $X$ will denote a metrizable locally convex topological vector space (LCS) with generating metric $d$; the terms $(X, d)$ and ( $X$, weak) refer to the topology under consideration. $X^{\prime}$ is the topological dual of $X$. In contrast to the preceding sections, BAIRE $(X, d)$ and $\operatorname{BAIRE}(X$, weak) denote the corresponding Baire $\sigma$-fields; similarly, $\operatorname{BOREL}(X, d)$ (respectively $\operatorname{BOREL}(X$, weak)) will denote the Borel $\sigma$-field of $(X, d)$ (respectively $(X$, weak)).

A function $\varphi$ from the measurable space $(\Omega, \Sigma)$ into $X$ is called Bochner measurable if it is the pointwise $d$-limit of a sequence of countably $X$-valued measurable functions on $\boldsymbol{\Omega}$. Clearly such a function is measurable w.r.t. $\operatorname{BOREL}(X, d), \varphi$ is called scalarly measurable if $x^{\prime} \circ \varphi$ is measurable for all $x^{\prime} \in X^{\prime}$. According to Edgar [5, Theorem 2.3], $\varphi$ is scalarly measurable if and only if it is measurable w.r.t. BAIRE $\left(X^{\prime}\right.$, weak), if and only if there is a sequence $\left(x_{n}^{\prime}\right)$ in $X^{\prime}$ such that, for any weakly Baire measurable map $\psi: \Omega \rightarrow X$ with $x_{n}^{\prime} \circ \psi=x_{n}^{\prime} \circ \varphi$ for all $n$, we have $\psi=\varphi$.

We now characterize $X$-valued strongly lifting compact maps.

4.1. TheOREM. Let $(X, d)$ be a metrizable LCS, $(\Omega, \Sigma, \nu)$ a complete probability space, and $\varphi: \Omega \rightarrow X$ a scalarly measurable function with image measure $\mu=\varphi(\nu)$ on BAIRE $(X$, weak). Then the following assertions are all equivalent:

(i) $\varphi$ is strongly lifting compact;

(ii) every lifting of $\mathscr{L}^{\infty}(\mu)$ is almost strong;

(iii) there is an almost strong lifting of $\mathscr{L}^{\infty}(\mu)$;

(iv) ( $X, \operatorname{BAIRE}(X$, weak), $\mu$ ) is completion regular, and $\mu$ is $\tau$-additive;

(v) there is a $\mu$-measurable closed linear subspace of $X$ which supports $\mu$ and is separable with respect to $d$;

(vi) every Borel subset of $(X, d)$ is measurable w.r.t. $\mu$, and $\mu$ is $\tau$-additive;

(vii) $\varphi$ agrees $\mu$-a.e. with a Bochner measurable function. 
Proof. (i) $\Rightarrow$ (ii) follows from 2.3.

(ii) $\Rightarrow$ (iii) is trivial.

(iii) $\Rightarrow$ (iv) was proved in [2].

(iv) $\Rightarrow$ (v). By a result of Tortrat [13, Theorem 8], $\mu$ is supported by a $d$-separable closed linear subspace; hence the implication follows.

(v) $\Rightarrow$ (vi). Let $Y$ be a $\mu$-measurable, $d$-separable closed linear subspace of $X$ supporting $\mu$. Then $\operatorname{BAIRE}(Y$, weak $)=\operatorname{BOREL}(Y, d)$, and $\mu$ is $\tau$-additive because $Y$ is Lindelöf. If $A \in \operatorname{BOREL}(X, d)$, then $A=(A \backslash Y) \cup(A \cap Y)$, $\mu(A \backslash Y)=0$, and $A \cap Y$ is $\mu$-measurable.

(vi) $\Rightarrow$ (v) follows from Tortrat [13, Theorem 8].

(v) $\Rightarrow$ (vii). Let $Y$ be a $\mu$-measurable, $d$-separable closed linear subspace of $X$ supporting $\mu$, and let $\left(y_{n}\right)$ be dense in $Y$. If $\Omega_{0}:=\varphi^{-1}(Y)$, then $\Omega_{0} \in \Sigma$ and $\nu\left(\Omega_{0}\right)=1$. For every $k, n \in \mathbb{N}$ put

$$
\Omega_{n}^{k}:=\varphi^{-1}\left(Y \cap\left\{y \in Y \mid d\left(y, y_{n}\right) \leqslant \frac{1}{k}\right\}\right) \backslash \bigcup_{m<n} \Omega_{m}^{k} .
$$

If $\varphi_{k}: \Omega \rightarrow X$ is defined by

$$
\begin{array}{ll}
\varphi_{k}(\omega)=y_{n} & \text { for } \omega \in \Omega_{n}^{k}, \\
\varphi_{k}(\omega)=y_{1} & \text { for } \omega \in \Omega \backslash \Omega_{0},
\end{array}
$$

then we see that $\varphi_{n}$ converges to $\varphi$ on $\Omega_{0}$.

(vii) $\Rightarrow$ (i). Let $\Omega_{0} \in \Sigma$ with $\nu\left(\Omega_{0}\right)=1$ be such that $\left.\varphi\right|_{\Omega_{0}}$ is Bochner measurable. Since $\varphi\left(\Omega_{0}\right)$ is $d$-separable, we can find a sequence $\left(x_{n}^{\prime}\right)$ in $X^{\prime}$ which separates the points of $\mathrm{cl}_{X}\left(\varphi\left(\Omega_{0}\right)\right)$. Hence Theorem 2.2 applies.

4.2. Remark. From Theorem 4.1 we immediately deduce that for any Baire probability $\mu$ on ( $X$, weak) the following is true:

If, among all maps $\varphi$ from a complete probability space $(\Omega, \Sigma, \nu)$ into $X$ such that $\mu$ is the image of $\nu$ under $\varphi$, there is one which is strongly lifting compact, then all these maps are strongly lifting compact. In particular, the identity map on $X$ then agrees $\mu$-a.e. with a Bochner measurable map.

4.3. ThEOREM. Let $(X, d)$ be a metrizable LCS. Then the following assertions are all equivalent:

(i) ( $X$, weak) is strongly lifting compact;

(ii) For every Baire probability measure $\mu$ on (X, weak), every lifting of $\mathscr{L}^{\infty}(\mu)$ is almost strong;

(iii) For every Baire probability measure $\mu$ on ( $X$, weak), there is an almost strong lifting of $\mathscr{L}^{\infty}(\mu)$;

(iv) ( $X$, weak) is completion regular and measure compact; 
(v) Every Baire probability measure $\mu$ on ( $X$, weak) is supported by a $\mu$-measurable closed linear subspace of $X$ which is separable with respect to $d$;

(vi) ( $X$, weak) is measure compact, and every Borel subset of $(X, d)$ is measurable with respect to any Baire probability measure on ( $X$, weak);

(vii) Every scalarly measurable function $\varphi$ from a complete probability space $(\Omega, \Sigma, \nu)$ into $X$ agrees $\nu$-a.e. with a Bochner measurable function.

The proof of this theorem is obvious from Theorem 4.1 and will be omitted. In order to state an easy corollary, we have to introduce some further notions.

4.3. Definition. Let $\left(T, \mathscr{B}_{T}, \mu\right)$ be a Baire probability space. Then $\left(T, \mathscr{B}_{T}, \mu\right)$ is said to have the almost strict disintegration property, if the following is true: $\mu$ is tight, and for every Lusin measurable map $\varphi$ from a probability space $\left(S, \mathscr{B}_{S}, \nu\right)$ into $T$, there is a map $t \rightarrow \lambda_{t}$ satisfying the conditions

(i) $\lambda_{t}$ is a tight probability measure on $\left(S, \mathscr{B}_{S}\right)$ for all $t \in T$;

(ii) for every $g \in \mathscr{C}^{b}(S)$, the function $t \mapsto \int g d \lambda_{t}$ is $\mu$-measurable;

(iii) for every $g \in \mathscr{C}^{b}(S)$ and $h \in \mathscr{C}^{b}(T)$, we have

$$
\int(h \circ \varphi) g d \nu=\int h(t) \int g d \lambda_{t} d \mu(t)
$$

(iv) $\operatorname{supp} \lambda_{t} \subset \varphi^{-1}(\{t\})$ for $\mu$-a.a. $t \in T$.

The following is a straightforward generalization of the results of A. and C. Ionescu Tulcea [8, Chapter 9, Section 2, Theorem 3, and Section 5, Theorem 5].

4.4. LEMMA. The Baire probability space $\left(T, \mathscr{B}_{T}, \mu\right)$ has the almost strict disintegration property if and only if $\mu$ is tight and $\mathscr{L}^{\infty}(\mu)$ has an almost strong lifting.

The proof of this lemma uses essentially the same ideas as in [8] and will not be given here.

4.5. Corollary. Suppose that $(X, d)$ is complete or that ( $X$, weak) is strongly measure compact. Then ( $X$, weak) is strongly lifting compact if and only if $(X, \operatorname{BAIRE}(X$, weak $), \mu)$ has the almost strict disintegration property for every Baire probability $\mu$ on ( $X$, weak).

Proof. If ( $X$, weak) is strongly lifting compact, then every Baire measure $\mu$ on ( $X$, weak) is $\tau$-additive. By Tortrat's theorem [13, Theorem 8], $\mu$ lives on a separable closed linear subspace $Y$ of $X$ which is a polish space if $(X, d)$ is complete. Since on $Y, \operatorname{BOREL}(Y, d)=\operatorname{BAIRE}(Y$, weak) we deduce that $\mu$ is tight. So in either case, $\mu$ is a tight probability, and hence the statement of the corollary follows at once from Theorem 4.3 (iii). 
4.6. Remark. Analogously one can show that, under the same assumptions, a scalarly measurable $X$-valued map is strongly lifting compact if and only if the induced probability space on $X$ has the almost strict disintegration property.

Strong lifting compactness of $X$ can be characterized in a purely topological way, as we shall show below.

4.7. THEOREM. Let $(X, d)$ be a metrizable LCS. Then the following assertions are all equivalent:

(i) ( $X$, weak) is strongly lifting compact;

(ii) ( $X$, weak) is measure compact, and $\{0\} \in \operatorname{BAIRE}(X$, weak);

(iii) ( $X$, weak) is measure compact, and there is a sequence in $X^{\prime}$ which separates the points of $X$;

(iv) ( $X$, weak) is measure compact, and there is a continuous linear injection from $(X, d)$ into $\mathbb{R}^{\mathbf{N}}$

(v) ( $X$, weak) is measure compact and submetrizable.

Proof. (i) $\Rightarrow$ (ii). Let $\mu$ be a Baire probability on ( $X$, weak). By Theorem 4.3 (v), there is a Baire set $B$ in $X$ with $\mu(B)=1$ whose closed linear span $Y$ is $d$-separable. Since $B \in \operatorname{BOREL}(Y, d)=\operatorname{BAIRE}(Y$, weak), it follows that $\{x\} \in$ $\operatorname{BAIRE}(X$, weak) for every $x \in B$. By translation invariance of $\operatorname{BAIRE}(X$, weak), we then have $\{0\} \in \operatorname{BAIRE}(X$, weak).

(ii) $\Rightarrow$ (iii) follows from Edgar's result cited in the beginning of this section.

(iii) $\Rightarrow$ (iv). Let $\left(x_{n}^{\prime}\right)$ be a sequence in $X^{\prime}$ which separates the points of $X$ and define a map

$$
\Phi: X \rightarrow \mathbb{R}^{\mathbf{N}}
$$

by

$$
\Phi(x)=\left(x_{n}^{\prime}(x)\right), \quad x \in X
$$

Then $\Phi: X \rightarrow \mathbb{R}^{\mathbf{N}}$ is an injection from $X$ into $\mathbb{R}^{\mathbf{N}}$, as desired.

(iv) $\Rightarrow$ (v). If $\Phi: X \rightarrow \mathbb{R}^{\mathbf{N}}$ is a linear injection, continuous with respect to $d$, then it is obviously continuous as a map from $\left(X\right.$, weak) into $\mathbb{R}^{N}$.

Let $d_{1}$ be a continuous metric on $\mathbf{R}^{\mathbf{N}}$. Then the composition with $\Phi$ produces a continuous metric on ( $X$, weak).

(v) $\Rightarrow$ (i) follows from Remark 3.2(iii).

4.8. CoROllarY. If the LCS $X$ is normable, then ( $X$, weak) is strongly lifting compact if and only if it is measure compact, and there is a continuous linear injection from $\left(X\right.$, norm) into $l^{\infty}(\mathbb{N})$. 
Proof. This follows from Theorem 4.7, provided tht the sequence $\left(x_{n}^{\prime}\right)$ in (iii) is chosen in such a way that it consists of elements of norm 1, which is always possible.

4.9. Remarks. (i) The injection of $X$ into $\mathbb{R}^{\mathbf{N}}$, or into $l^{\infty}(\mathbb{N})$, in statement (iv) of Theorem 4.7, or in Corollary 4.8, respectively, is not an isomorphism in general or even an isometry. For instance, the natural injection from $l^{2}(\mathbb{N})$ into $l^{\infty}(\mathbb{N})$ given by the identity map is a continuous map without continuous inverse.

(ii) From the proof of Theorem 4.7 it follows at once that, in order to show that the measure compact space $X$ is strongly lifting compact, it suffices to check that there is one $X$-valued strongly lifting compact map.

4.10. EXAMPLEs. (i) The space $l^{\infty}(\mathbb{N})$ is submetrizable under its weak topology, but it is not strongly lifting compact since it is not measure compact (see Edgar [6, Section 5, page 575]).

(ii) Obviously, $l^{p}(\mathbf{N})(1 \leqslant p<\infty)$ is strongly lifting compact because it is a separable metrizable linear space, whereas $l^{2}[0,1]$ is not.

(iii) Let $X=M[0,1]$ be the dual space of $\mathscr{C}^{b}[0,1]$. Then $X$ is a non-separable Banach space. Clearly the weak topology of $X$ is submetrizable. Under the mild assumption that the continuum be measure compact, it can be deduced that ( $X$, weak) is measure compact (see Edgar [6, Corollary 3.5]). Hence, by Theorem 4.7(v), we obtained an example of a non-separable strongly lifting compact space.

4.11. REMARK. Let $X$ be an LCS, $(\Omega, \Sigma, \nu)$ a complete probability space, and $\varphi$ a Baire measurable map from $(\Omega, \Sigma)$ into $(X$, weak) such that, for every $n \in \mathbb{N}$, there is $\Omega_{n} \in \Sigma$ with $\nu\left(\Omega_{n}\right) \geqslant 1-1 / n$ whose image under $\varphi$ is contained in a $\varphi(\nu)$-measurable hull of a bounded subset $B_{n}$ of $X$. If $\varphi$ is any bounded linear lifting of $\mathscr{L}^{\infty}(\nu)$, then a Baire measurable map $\rho^{\prime}(\varphi)$ from $\left(\Omega_{0}, \Sigma \cap \Omega_{0}\right)$ into the strong bidual $X^{\prime \prime}$ of $X$ in its weak topology $\sigma\left(X^{\prime \prime}, X^{\prime}\right)$ may be defined by

$$
x^{\prime} \circ \rho^{\prime}(\varphi)(\omega):=\rho\left(\chi_{\Omega_{n}^{\prime}} \cdot x^{\prime} \circ \varphi\right)(\omega), \quad \omega \in \Omega_{n}^{\prime},
$$

where $\Omega_{n}^{\prime}:=\rho\left(\Omega_{n}\right) \backslash \bigcup_{m<n} \Omega_{m}^{\prime}$ and $\Omega_{0}:=\cup \Omega_{n}^{\prime}$. (Clearly $\rho(A)=\left\{\omega \mid \rho\left(\chi_{A}\right)(\omega)=\right.$ $1\}$, for any $A \in \Sigma$.)

Let us say that $\varphi$ is strongly linearly lifting compact if $\varphi(\omega)=\rho^{\prime}(\varphi)(\omega) \nu$-a.e. It can be seen that Theorem 4.1 (and Theorem 4.2, Corollary 4.5, Theorem 4.7, Corollary 4.8 as well) remain true if the term 'strongly lifting compact' is replaced by the term 'strongly linearly lifting compact' throughout. Details will appear elsewhere. 


\section{References}

[1] A. G. A. G. Babiker, G. Heller and W. Strauss, 'On a lifting invariance problem' (in Measure Theory, Oberwolfach 1983 Proceedings, Springer Lecture Notes 1089).

[2] A. G. A. G. Babiker and W. Strauss, 'Almost strong liftings and $\tau$-additivity' (in Measure Theory, Oberwolfach 1977 Proceedings, Springer Lecture Notes 695).

[3] A. Bellow, 'Lifting compact spaces' (in Measure Theory, Oberwolfach 1979 Proceedings, Springer Lecture Notes 794).

[4] A. Bellow, 'Mesures de Radon et espaces relèvement compacts', C.R. Acad. Sci. Paris Sér. A 289 (1979), 621-624.

[5] G. A. Edgar, 'Measurability in a Banach space', Indiana Univ. Math. J. 26 (1977), 663-667.

[6] G. A. Edgar, 'Measurability in a Banach space II', Indiana Univ. Math. J. 28 (1979), 559-579.

[7] G. A. Edgar and M. Talagrand, 'Liftings of functions with values in a completely regular space', Proc. Amer. Math. Soc. 78 (1980), 345-349.

[8] A. and C. Ionescu-Tulcea, Topics in the theory of lifting (Springer, 1969).

[9] A. Ionescu-Tulcea, 'On pointwise convergence, compactness, and equicontinuity II', Advances in Math. 12 (1974), 171-177.

[10] J. D. Knowles, 'Measures on topological spaces', Proc. London Math. Soc. 17 (1967), 139-156.

[11] V. Losert, 'A measure space without the strong lifting property', Math. Ann. 239 (1979), 119-128.

[12] W. Moran, 'Measures and mappings on topological spaces', Proc. London Math. Soc. 19 (1969), 493-508.

[13] A. Tortrat, 'Prolongements $\tau$-réguliers, applications aux probabilités Gaussiennes', Symposia Mathematica, Vol. XXI (convegno sulle Misure su Gruppi e su spazi Vettoriali,...), INDAM, Rome 1975, 117-138.

[14] V. S. Varadarajan, 'Measures on topological spaces', Math. Sbornik 55 (1961), 33-100 (Russian), Amer. Math. Soc. Transl. 48 (1965), 141-228.

School of Mathematics

The University of Khartoum

P.O. Box 321

Khartoum

Sudan

Mathematisches Institut A

Universität Stuttgart

Pfaffenwaldring 57

D 7000 Stuttgart

West Germany
Mathematisches Institut II

Universität Karlsruhe

Englerstrasse 2

D 7500 Karlsruhe West Germany 